\title{
La cura: una questione di merito?
}

\author{
Sandro Spinsanti \\ Istituto Giano per le Medical Humanities, Riano, Roma - Italia
}

\begin{abstract}
The cure: is it a question of merit?
The SIAARTI document "Recommendations of clinical ethics for admission to intensive treatments and for their suspension" has stimulated mixed reactions. The "tragic decision" which clinicians may be required to take emerge in the background: which patients to accept and which to reject in intensive care units, which the epidemic has revealed to be less than required. Particularly, clinicians are invited to reflect on the concept of responsibility in choices. With regards to an ethical responsibility we are facing a medico-legal interpretation, that is translated into the explanation of the criteria with which choices are made and the assumption of their moral weight. This scenario of tragic choices confirms the intuition contained in the mythological figure of the "wounded healer".
\end{abstract}

Keywords: Admission to treatment, COVID-19, Customization of choices, Ethical responsibility, Resource allocation

Un documento pubblicato dall'associazione dei medici anestesisti e rianimatori (SIAARTI) (1) sta suscitando un animato dibattito, attraversato da profondi timori. Si tratta di raccomandazioni rivolte ai colleghi dai quali dipende se avviare o meno a trattamenti intensivi i malati che ne hanno bisogno per sopravvivere. In concreto, il riferimento è ai malati ai quali l'epidemia di COVID-19 ha compromesso le capacità respiratorie. Le condizioni eccezionali nelle quali l'epidemia si sta sviluppando ci costringe a confrontarci con i limiti delle risorse disponibili. In termini crudi: la capacità delle rianimazioni sta raggiungendo il limite; una volta saturate, i medici dovranno decidere chi ammettere e chi escludere. Chi è destinato a sopravvivere?

In uno scenario di servizi sanitari privatizzati, diremmo cinicamente: la differenza tra i sommersi e i salvati la farà il mercato. Sopravvivrà chi ha i mezzi e le coperture assicurative. Per fortuna, il servizio sanitario pubblico, a base universalistica, ci protegge da questo incubo. Rimane, però, la necessità di un criterio per fare le scelte, quando la domanda eccede la disponibilità. Ci rifugeremo nel criterio dei "primi arrivati"? Anche questa ipotesi è inquietante. Qui si inseriscono i suggerimenti in questione rivolti ad anestesisti e rianimatori. II criterio dei limiti di età ha evocato il fantasma

Received: March 29, 2020

Accepted: April 8, 2020

Published online: May 2, 2020

Indirizzo per la corrispondenza:

Sandro Spinsanti

Istituto Giano

Via Stazzo Quadro 7

00060 Riano, Roma - Italia

Sandro.spinsanti@gmail.com dei vecchi automaticamente esclusi. La "massimizzazione dei benefici per il maggior numero di persone" ha fatto emergere, analogamente, il criterio di scelte fatte sulla base della quantità di vita a scapito di altre valutazioni riferite alla persona.

La prospettiva della personalizzazione delle scelte evoca un antico problema: il medico è autorizzato a decidere chi merita di essere salvato? II drammaturgo Bernard Shaw ha avuto il coraggio di portarlo a teatro, nel 1906, con // dilemma del dottore. Immagina che il protagonista abbia inventato una nuova cura per i malati di tisi, ma ha una disponibilità limitata: non più di dieci posti. Una giovane signora gli chiede di prendere in cura suo marito, che è un artista famoso. La prima considerazione del dottore è brutale: "Non capita tutti i giorni di trovare una persona che meriti davvero di essere salvata. Dovrò far uscire un altro dall'ospedale, ma troverò sicuramente qualcuno peggiore di lui". Salvo poi ricredersi, considerando che la signora è più interessante per lui come vedova...

Ecco la peggiore delle insidie: far dipendere la scelta del medico dalla qualità morale, vera o presunta, della persona malata. Ciò è, fortunatamente, escluso dalla deontologia professionale dei medici. Non abbiamo, con ciò, messo a fuoco quali possano o debbano essere i criteri positivi in base ai quali prendere le decisioni cliniche nei contesti di risorse insufficienti che obbligano a operare delle scelte. Forse, in questo ambito, possiamo, per il momento, proclamare, negativamente, "ciò che non siamo, ciò che non vogliamo" (Eugenio Montale): non possiamo accettare che la cura sia legata al merito, stabilendo una gerarchia tra persone più o meno degne.

Tenendo stretto questo imprescindibile criterio deontologico, siamo costretti ad affacciarci, volenti o nolenti, nel territorio dell'etica. Oltre allo scenario delle scelte "corrette", qui 
ci domandiamo quali rispondono ai criteri etici di decisioni "buone" o "giuste". L'epidemia minaccia dalle fondamenta un edificio che, con grande fatica, da qualche tempo ci stavamo sforzando di costruire. All'alba del nuovo millennio, un gruppo di professionisti e di studiosi, per iniziativa dell'European Federation of Internal Medicine e dell'American College of Physicians, ha proposto la "Carta della professionalità medica", pubblicata nel 2002 da The Lancet e Annals of Internal Medicine (2). Innovando la tradizione secolare dell'etica medica, che faceva coincidere la buona medicina con quella che assicura il bene del paziente, deciso dal medico in scienza e coscienza, la Carta individuava tre criteri con i quali le decisioni mediche si dovevano misurare: fornire cure efficaci, secondo lo standard delle conoscenze mediche, rispettare il paziente come persona autonoma, tenendo in considerazione le sue scelte e le sue preferenze, e garantire a tutti i cittadini uguali opportunità, con equità e senza discriminazioni. I tre criteri, sorretti rispettivamente dai principi di beneficità, autonomia e giustizia, dovevano essere tenuti in considerazione contemporaneamente. L'etica medica passava, dopo una riproposta costante durata secoli, da una a tre dimensioni.

Il movimento di Slow Medicine è riuscito a sintetizzare questo cambio di paradigma proponendo cure "sobrie-rispettose-giuste", ovvero cure appropriate, sulla base dei migliori studi di efficacia, costruite mettendo insieme le indicazioni cliniche con la biografia della persona malata e, quindi, sollecitando un suo consenso come espressione di consapevolezza; cure rivolte a tutti quelli che ne hanno diritto e bisogno, senza privilegi. Ebbene, questa costruzione, insieme concettuale e pratica, minaccia di essere spazzata via dall'emergenza. Mancano le risorse (posti letto, ausili, farmaci, per non parlare di un sufficiente numero di professionisti) e di coinvolgimento del malato nelle scelte non si sente parlare, neppure nella forma estremamente riduttiva del consenso informato; l'equità si dilegua sotto la spinta del "triage". Certo, "à la guerre comme à la guerre"... Ma l'etica della cura in tempo di pace è diversa. Con l'auspicio del ritorno della normalità, non possiamo che desiderare il ritorno a cure "sobrie-rispettose-giuste".

\section{Disclosures}

Conflict of interest: The author declares no conflict of interest. Financial support: The author declares no financial support.

\section{Bibliografia}

1. Raccomandazioni di etica clinica per l'ammissione a trattamenti intensivi e per la loro sospensione, in condizioni eccezionali di squilibrio tra necessità e risorse disponibili. http://www.siaarti. it/SiteAssets/News/COVID19\%20-\%20documenti\%20SIAARTI/ SIAARTI\%20-\%20Covid19\%20-\%20Raccomandazioni\%20di\%20 etica\%20clinica.pdf.

2. https://educazioneprofessionale.cdl.unimi.it/sites/ld72/files/ 2019-05/Carta_professio_medica.pdf. 